\title{
On the integration of an axially symmetric galaxy model
}

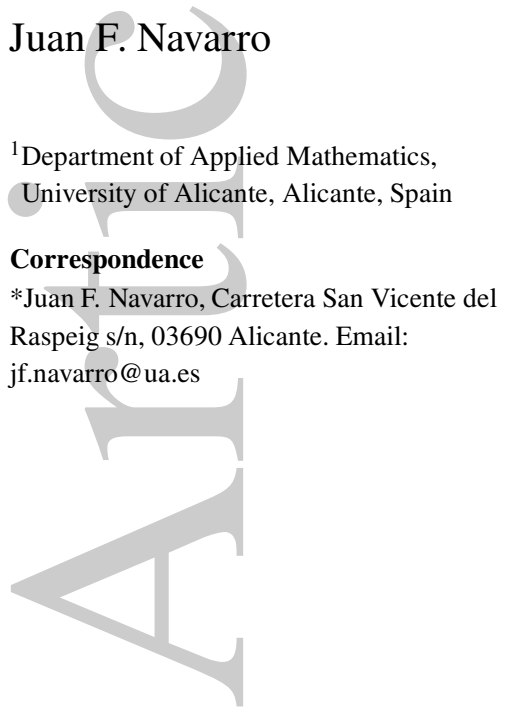

\section{Summary}

A numerical exploration of the escape of a particle from a potential well modeling an axially symmetric galaxy requires the integration of millions of orbits over very long spans of time. In this paper, we propose an integration of the equations of motion of the system by recurrent power series. We prove the convergence of the method, compare the computational time of the recurrent series method against the DormandPrince method, and study the propagation of the truncation errors in the numerical solutions.

\section{KEYWORDS:}

Galactic potentials, Recurrent power series, Escapes, Hamiltonian systems

\section{I INTRODUCTION}

Over the last decades, the subject of escaping particles from an open dynamical system has been an active field of research $([1,2,3,4,5,6,7,8,9,10,11,12,13])$. Contopoulos [6] explored this phenomenon in Hamiltonian systems representing the central part of perturbed galaxies with quadruple symmetry and four openings to infinity, founding that the openings of the potential well are bridged by unstable periodic orbits. The asymptotic curves of these orbits govern the escape to infinity from the potential well. Later, Navarro and Henrard [10] analyzed the shapes and sizes of the windows of escape of stars from the simplified galactic model studied by Contopoulos, finding that they consist of a main window and of a hierarchy of secondary windows with an intricate spiral structure. In 2017, Navarro [11] investigated the shape of the windows of escape to infinity from a galaxy modeled by a bi-symmetrical two-dimensional harmonic oscillator with quartic perturbing terms, finding the same mechanism governing the escape from the potential well.

In 2012, Zotos [12] studied the behavior of orbits in a time-independent axially symmetric galactic-type potential, presenting only one opening to infinity. This model describes the motion of a particle in the central part of a galaxy, for values of the energy larger than the escape energy.

The study of the escape of a particle from the potential well of a dynamical system requires an accurate integration of the equations of motion for long spans of time. This fact forces to perform an analysis of the accumulation of the truncation errors in the numerical solutions if we are interested in accurate and reliable numerical solutions.

In 1965, Deprit and Price [14] adapted the ideas of Steffensen [15] to the canonical equations of motion of the planar restricted problem of three bodies in the barycentric synodical coordinate system. In their work, Deprit and Price replaced a fourth-order system by a system of eight differential equations, all of first order. They followed Steffensen to prove that the power series computed by recurrence are convergent for any set of initial conditions, provided it does not belong to the phase planes of binary collisions. The method proposed by Steffensen was much more accurate and achieved the smallest CPU-times compared to classical numerical integration methods $[14,16]$.

Navarro [17] followed the ideas of Deprit and Price [14] and Steffensen [15] to integrate the equations of motion of the $N$-body ring problem by replacing the the fourth-order system of differential equations by a system of $6+2 N$ first order differential equations. Navarro showed that the resulting system is integrable by means of recurrent power series, and obtained

This article has been accepted for publication and undergone full peer review but has not been through the copyediting, typesetting, pagination and proofreading process, which may lead to differences between this version and the Version of Record. Please cite this article as doi: $10.1002 / \mathrm{cmm} 4.1062$ 
the convergence ratio of the series. He also proved the superiority of the recurrent power series method over more classical methods, like the Runge-Kutta of order 4, the Runge-Kutta-Fehlberg method (with constant and variable step size) and the Dormand-Prince method (with constant and variable step size) [18].

The aim of this paper is to present a numerical method for the integration of the equations of motion of a a time-independent axially symmetric galactic-type potential, presenting only one opening to infinity, by recurrent power series. We prove the convergence of the recurrent power series and compare the integration by this method against the Dormand-Prince method. We include in this analysis the study of the propagation of the truncation errors in the numerical solutions.

\section{I THE EQUATIONS OF MOTION}

We are interested in the integration of the equations of motion of a system modeled by the potential

$$
V(r, z)=\frac{\omega^{2}}{2}\left(r^{2}+z^{2}\right)-\mu\left(\alpha\left(r^{4}+z^{4}\right)+2 \rho r^{2} z^{2}\right) .
$$

Potentials made up of perturbed harmonic oscillators, are among the most well studied in non-linear dynamics ([12, 19, 20, 21]). This potential can be derived by expanding global galactic potentials near the central stable equilibrium point of the system, that is, the center of the galaxy, and it describes the local motion in the meridian $(r, z)$ plane near the central part of an axially symmetric galaxy. In (11), $r$ and $z$ are the usual cylindrical coordinates, and $\omega, \mu, \alpha$ and $\rho$ are parameters. Such galactic potentials arise if the density distribution near the galactic center is an analytic function of the coordinates and the Taylor series for the corresponding potential is truncated at fourth order. Here, we must stress that this galactic type potential is truncated at $r_{\max }=1.5$ $\mathrm{kpc}$, that is, the present model can only describe local motion at small distances from the galactic center $\left(r_{\max } \leq 1.5 \mathrm{kpc}\right)$. Otherwise, the mass density increases outwards from the center, which is practically never observed in galaxies. Therefore, we study the phenomenon of escape of stars from the central part of a galaxy.

As the potential $V(r, z)$ is axially symmetric and the $L_{z}$ component of the angular momentum is conserved, the dynamical structure of the galactic system can be investigated using the effective potential

$$
W(r, z)=-\frac{L_{z}^{2}}{2 r^{2}}-V(r, z)
$$

in order to study the properties of motion in the meridian $(r, z)$ plane. The Hamiltonian corresponding to potential (2) reads

that is

$$
H=\frac{1}{2}\left(\dot{r}^{2}+\dot{z}^{2}\right)-W(r, z),
$$

$$
H=\frac{1}{2}\left(\dot{r}^{2}+\dot{z}^{2}\right)+\frac{\omega^{2}}{2}\left(r^{2}+z^{2}\right)-\mu\left(\alpha\left(r^{4}+z^{4}\right)+2 \rho r^{2} z^{2}\right)+\frac{L_{z}^{2}}{2 r^{2}} .
$$

In this formulation, we have used a system of galactic units, taking $1 \mathrm{kpc}$ as the unit of length and $10^{7} \mathrm{yr}$ as the unit of time. The velocity unit is $1 \mathrm{kpc}\left(10^{7} \mathrm{yr}\right)^{-1}=97.8 \mathrm{~km} \mathrm{~s}^{-1}$ and the energy unit is $1 \mathrm{kpc}^{2}\left(10^{7} \mathrm{yr}\right)^{-2}$. In our analysis, we will consider the following values of the parameters of the system: $\omega=1\left(10^{7} \mathrm{yr}\right)^{-1}, \mu=1\left(10^{7} \mathrm{yr} \mathrm{kpc}\right)^{-2}, \alpha=0.2$ and $\rho=-1.2$.

There is a value of the energy $\left(H_{c}\right)$ such that, for values of $H$ larger than $H_{c}$, the potential well opens up to infinity and test particles may escape. For each larger value of $H$, there is an unstable periodic orbit across the opening, bouncing back and forth between the two "walls" of the pass. Orbits that cross the Lyapunov orbit with velocity outwards will escape from the potential well.

\section{I CURVES OF ZERO VELOCITY}

The curves of zero velocity of the Hamiltonian system (3) are given by the relation

$$
f\left(r^{2}, z^{2}\right)=H+W\left(r^{2}, z^{2}\right)=0 .
$$

The points of the $r-z$ plane where the curves open are the saddle-points of (5). In the case $L_{z}=0$, the energy of escape for the potential (2) can be found theoretically by the procedure followed by Caranicolas [19], and is given by

$$
H_{c}=\frac{1}{16 \mu \alpha} \text {. }
$$

This article is protected by copyright. All rights reserved. 
In the general case $\left(L_{z} \neq 0\right)$, the value of the energy of escape can be computed numerically. Zotos [12] found the following relation between $H_{c}$ and $L_{z}$,

$$
L_{z}=-55738.3 H_{c}^{4}+78897.2 H_{c}^{3}-41862.2 H_{c}^{2}+9871.87 H_{c}-872.985 .
$$

The curves of zero velocity are the curves defined by

$$
H=\frac{L_{z}^{2}}{2 r^{2}}+\frac{\omega^{2}}{2}\left(r^{2}+z^{2}\right)-\mu\left(\alpha\left(r^{4}+z^{4}\right)+2 \rho r^{2} z^{2}\right)
$$

The critical value of the energy associated to the values of the parameters given by $\omega=1, \mu=1, \alpha=0.2, \rho=-1.2$ and $L_{z}=0.1$, is $H_{c}=0.3125$. For each larger value of $H$, there is an unstable periodic orbit across the opening, bouncing back and forth between the two "walls" of the pass (see right panel of Figure2).

In Figure1, we show the relation between $L_{z}$ and the energy of escape $H_{c}$. The gray region corresponds to values of $\left(H, L_{z}\right)$ for open curves of zero velocity. In the left panel of Figure 2, we show the curves of zero velocity of the system for $L_{z}=0.1$ and $H_{1}=0.32, H_{2}=0.36, H_{3}=0.4$. In the right panel, we represent the curve of zero velocity for $L_{z}=0.1$ and $H=0.32$, and the corresponding Lyapunov periodic orbit "guarding" the opening in the potential well.

A typical orbit starting somewhere inside the potential well will first bounce around in it and then eventually leave the region through the open of the potential well. Due to the chaotic character of the system, the sensitive dependence to the initial conditions make not possible to make neither exact nor approximative predictions. In Figure 3, we show some orbits of the system with different escaping times for $\omega=1, \mu=1, \alpha=0.2, \rho=-1.2$ and $L_{z}=0.1$.

\section{I NUMERICAL INTEGRATION OF THE EQUATIONS OF MOTION}

The canonical equations of motion described by Hamiltonian (3) are given by

$$
\begin{aligned}
& \dot{r}=p, \\
& \dot{z}=q, \\
& \ddot{r}=\dot{p}=\frac{\partial W}{\partial r}, \\
& \ddot{z}=\dot{q}=\frac{\partial W}{\partial z}, \\
& \dot{r}=p, \\
& \dot{z}=q, \\
& \dot{p}=-r+4 \alpha r^{3}+4 \rho r z^{2}+\frac{L_{z}^{2}}{r^{3}}, \\
& \dot{q}=-z+4 \alpha z^{3}+4 \rho r^{2} z .
\end{aligned}
$$

Here, we have assumed that $\omega=\mu=1$. The method proposed by Steffensen [15] is made practical by the introduction of a certain number of auxiliary dependent variables, which transform the system of differential equations of motion in a new system where all denominators have been removed, and only products of two dependent variables appear. This form is particularly well-prepared for the substitution of power series and the identification of equal powers in $t$. The application of the series is particularly interesting because the square roots are completely avoided in the computations, and the computation of all the coefficients of the power series is done in a recurrent way.

Let us introduce the quantity

$$
R=\frac{1}{r^{3}}
$$

The relation between the derivatives of $r$ and $R$ is obtained through

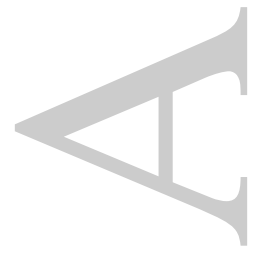

$$
r \dot{R}=-3 R \dot{r} .
$$

This article is protected by copyright. All rights reserved. 
Hence, the canonical equations (10) may be replaced by a system of five differential equations

$$
\begin{aligned}
\dot{r} & =p, \\
\dot{z} & =q, \\
r \dot{R} & =-3 R \dot{r}, \\
\dot{p} & =-r+4 \alpha r^{3}+4 \rho r z^{2}+L_{z}^{2} R, \\
\dot{q} & =-z+4 \alpha z^{3}+4 \rho r^{2} z,
\end{aligned}
$$

which lend themselves to an integration by recurrent power series. The formal series

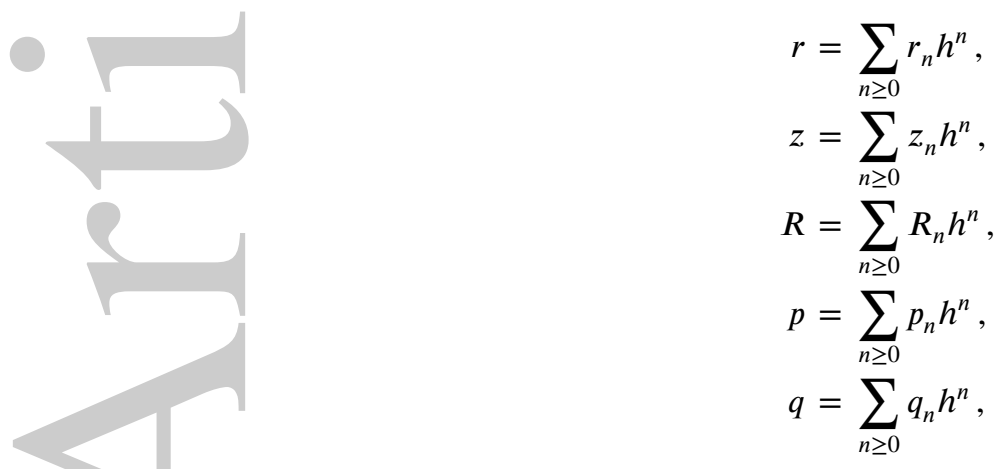

are introduced into the differential equations (13) and coefficients of $h^{n}$ are collected for each $n$. In this manner, for each $n$ one obtains the relations

$$
\begin{aligned}
(n+1) r_{n+1} & =p_{n}, \\
(n+1) z_{n+1} & =q_{n}, \\
(n+1) r_{0} R_{n+1} & =-\sum_{j=0}^{n-1}(j+1)\left(3 r_{j+1} R_{n-j}+r_{n-j} R_{j+1}\right)-3(n+1) r_{n+1} R_{0}, \\
U_{n} & =\sum_{j=0}^{n} r_{j} r_{n-j}, \\
V_{n} & =\sum_{j=0}^{n} z_{j} z_{n-j}, \\
(n+1) p_{n+1} & =-r_{n}+4 \alpha \sum_{j=0}^{n} r_{j} U_{n-j}+4 \rho \sum_{j=0}^{n} r_{j} V_{n-j}+L_{z}^{2} R_{n}, \\
(n+1) q_{n+1} & =-z_{n}+4 \alpha \sum_{j=0}^{n} z_{j} V_{n-j}+4 \rho \sum_{j=0}^{n} z_{j} U_{n-j} .
\end{aligned}
$$

Here, $U$ and $V$ are auxiliary quantities to ease the writing of the equations for $p_{n+1}$ and $q_{n+1}$. The values of $r, z, p$ and $q$ at the current integration time $t_{0}$ give

$$
r_{0}=r\left(t_{0}\right), \quad z_{0}=z\left(t_{0}\right), \quad p_{0}=\dot{r}\left(t_{0}\right), \quad q_{0}=\dot{z}\left(t_{0}\right),
$$

and the definition of the additional unknowns requires that

$$
U_{0}=r_{0}^{2}, \quad V_{0}=z_{0}^{2}, \quad R_{0}=\frac{1}{r_{0}^{3}}
$$

The series given in equation (14) are used to compute the value of the variables at time $t_{0}+h$, considering that $t_{0}$ is the current time of integration. The step size $h$ is chosen so that the solution have a desired accuracy. A detailed analysis on the determination of $h$ has been carried out by Sitarski [22].

\subsection{Convergence of the power series}

Theorem 1. The power series (14) are convergent, for any $r_{0} \neq 0$.

This article is protected by copyright. All rights reserved. 
Proof. In order to prove that, when $r_{0} \neq 0$, the power series are convergent, we introduce, following Deprit [14], and for every $n \geq 1$, the notation

and we show that, for every $n \geq 2$, the inequalities

$$
k_{n}=\frac{1}{n(n+1)},
$$

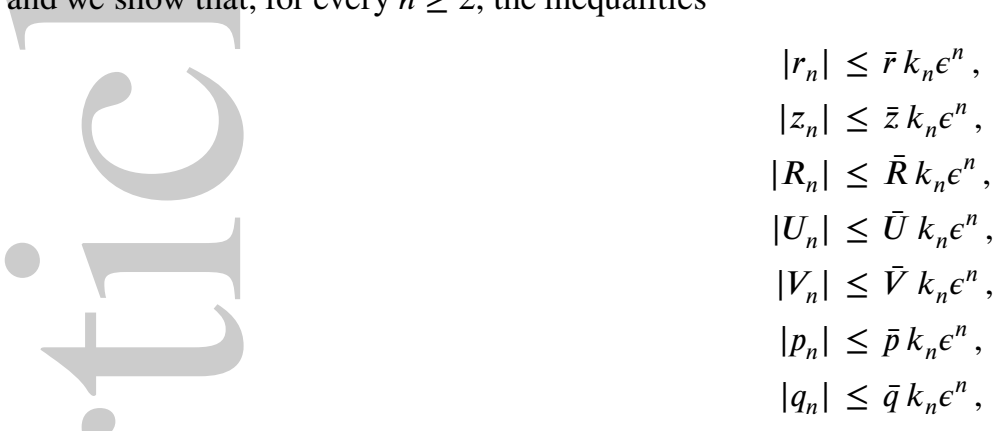

imply that

$$
\begin{aligned}
\left|r_{n+1}\right| & \leq \bar{r} k_{n+1} \epsilon^{n+1}, \\
\left|z_{n+1}\right| & \leq \bar{z} k_{n+1} \epsilon^{n+1}, \\
\left|R_{n+1}\right| & \leq \bar{R} k_{n+1} \epsilon^{n+1}, \\
\left|U_{n+1}\right| & \leq \bar{U} k_{n+1} \epsilon^{n+1}, \\
\left|V_{n+1}\right| & \leq \bar{V} k_{n+1} \epsilon^{n+1}, \\
\left|p_{n+1}\right| & \leq \bar{p} k_{n+1} \epsilon^{n+1}, \\
\left|q_{n+1}\right| & \leq \bar{q} k_{n+1} \epsilon^{n+1} .
\end{aligned}
$$

We obtain first, from equation [15],

$$
(n+1)\left|r_{n+1}\right|=\left|p_{n}\right| \leq \bar{p} k_{n} \epsilon^{n} .
$$

Hence, a sufficient condition for the vadility of (23) is

Since

$$
\bar{p} k_{n} \epsilon^{n} \leq(n+1) \bar{r} k_{n+1} \epsilon^{n+1} .
$$

for $n \geq 2$, the more rigid inequality

$$
\frac{k_{n}}{(n+1) k_{n+1}}=\frac{n+2}{n(n+1)}=\frac{1}{n+1}\left(1+\frac{2}{n}\right) \leq \frac{2}{3}
$$

$$
\frac{2}{3} \bar{p} \leq \epsilon \bar{r}
$$

is also a sufficient condition for the validity of (23). Following the same procedure with equation (16), we obtain the relation

$$
\frac{2}{3} \bar{q} \leq \epsilon \bar{z}
$$

as a sufficient condition for the validity of (24).

From equation (17), we get that

$$
(n+1)\left|R_{n+1}\right|\left|r_{0}\right| \leq 3(n+1)\left|r_{n+1}\right|\left|R_{0}\right|+\sum_{j=0}^{n-1}(j+1)\left(3\left|r_{j+1}\right|\left|R_{n-j}\right|+\left|R_{j+1}\right|\left|r_{n-j}\right|\right) .
$$

Now, taking into account inequalities (22) and (23), we obtain

$$
(n+1)\left|R_{n+1}\right|\left|r_{0}\right| \leq 3(n+1) \bar{r}\left|R_{0}\right| k_{n+1} \epsilon^{n+1}+4 \bar{r} \bar{R} \epsilon^{n+1} \sum_{j=0}^{n-1}(j+1) k_{j+1} k_{n-j} .
$$

Then, a sufficient condition for (25) is given by

$$
3(n+1) \bar{r}\left|R_{0}\right| k_{n+1}+4 \bar{r} \bar{R} \sum_{j=0}^{n-1}(j+1) k_{j+1} k_{n-j} \leq(n+1)\left|r_{0}\right| \bar{R} k_{n+1},
$$

This article is protected by copyright. All rights reserved. 
which leads to

$$
3 \bar{r}\left|R_{0}\right|+4(n+2) \bar{r} \bar{R} \sum_{j=0}^{n-1}(j+1) k_{j+1} k_{n-j} \leq\left|r_{0}\right| \bar{R} .
$$

Here, we have taken into account that

$$
\frac{1}{(n+1) k_{n+1}}=n+2 \text {. }
$$

From the relation

$$
n+2=(j+2)(n-j+1)-(j+1)(n-j),
$$

we deduce that

hence that

$$
(n+2) k_{j+1} k_{n-j}=\frac{1}{n+1}\left(\frac{1}{j+1}+\frac{1}{n-j}\right)-\frac{1}{n+3}\left(\frac{1}{j+2}+\frac{1}{n-j+1}\right),
$$

$$
(n+2) \sum_{j=0}^{n-1}(j+1) k_{j+1} k_{n-j}=\frac{n+2 \sigma_{n}}{n+3},
$$

where we have defined, for any $n \leq 1$,

$$
\sigma_{n}=\sum_{j=1}^{n} \frac{1}{j}
$$

However, for any $n \geq 2$,

$$
\begin{gathered}
\sigma_{n} \leq 1+\frac{1}{2}+\frac{1}{3}(n-2)=\frac{3}{2}+\frac{1}{3}(n-2), \\
n+2 \sigma_{n} \leq \frac{5}{3}(n+1),
\end{gathered}
$$

and

Thus, (25) is verified if

$$
\frac{n+2 \sigma_{n}}{n+3} \leq \frac{5}{3}\left(1-\frac{2}{n+3}\right) \leq \frac{5}{3} \text {. }
$$

Now, from equation (18), we can write

$$
3 \bar{r}\left|R_{0}\right| \leq\left(\left|r_{0}\right|-\frac{20}{3} \bar{r}\right) \bar{R} .
$$

$$
\left|U_{n+1}\right| \leq \sum_{j=0}^{n+1}\left|r_{j}\right|\left|r_{n+1-j}\right|=\left|r_{0}\right|\left|r_{n+1}\right|+\left|r_{n+1}\right|\left|r_{0}\right|+\sum_{j=1}^{n}\left|r_{j}\right|\left|r_{n+1-j}\right|,
$$

and the use of inequalities (22) and (23) yields

$$
\left|U_{n+1}\right| \leq 2\left|r_{0}\right| \bar{r} k_{n+1} \epsilon^{n+1}+\bar{r}^{2} \epsilon^{n+1} \sum_{j=1}^{n} k_{j} k_{n+1-j} .
$$

The identity

implies that

$$
n+2=(j+1)(n-j+2)-j(n-j+1)
$$

$$
(n+2) k_{j} k_{n-j}=\frac{1}{n+1}\left(\frac{1}{j}+\frac{1}{n-j+1}\right)-\frac{1}{n+3}\left(\frac{1}{j+1}+\frac{1}{n-j+2}\right)
$$

and then

$$
\sum_{j=1}^{n} k_{j} k_{n-j+1}=\frac{2\left(n+2 \sigma_{n}\right)}{(n+1)(n+2)(n+3)} \text {. }
$$

However,

$$
\sigma_{n} \leq n,
$$

which implies that

Here, we have used the relation

$$
\frac{2\left(n+2 \sigma_{n}\right)}{(n+1)(n+2)(n+3)} \leq 6 k_{n+1} \frac{n}{n+3}<6 k_{n+1} \text {. }
$$

Then, equation (35) can be written in the form

$$
k_{n+1}=\frac{1}{(n+1)(n+2)} \text {. }
$$

$$
\left|U_{n+1}\right| \leq 2\left|r_{0}\right| \bar{r} k_{n+1} \epsilon^{n+1}+6 \bar{r}^{2} k_{n+1} \epsilon^{n+1},
$$


and a sufficient condition for (27) reads

$$
2\left|r_{0}\right| \bar{r} k_{n+1}+6 \bar{r}^{2} k_{n+1} \leq \bar{U} k_{n+1}
$$

that is

$$
2\left|r_{0}\right| \bar{r}+6 \bar{r}^{2} \leq \bar{U}
$$

By following the same scheme and the same estimates as in the preceding case, we find that

$$
2\left|z_{0}\right| \bar{z}+6 \bar{z}^{2} \leq \bar{V} .
$$

is a sufficient condition for (28).

Finally, we examine (20). We give it the form

$$
(n+1) p_{n+1}=-r_{n}+L_{z}^{2} R_{n}+4 \alpha \sum_{j=0}^{n} r_{j} U_{n-j}+4 \rho \sum_{j=0}^{n} r_{j} V_{n-j} .
$$

Now it results convenient to write it as

$$
(n+1) p_{n+1}=-r_{n}+L_{z}^{2} R_{n}+4 \alpha\left(r_{0} U_{n}+r_{n} U_{0}\right)+4 \rho\left(r_{0} V_{n}+r_{n} V_{0}\right)+4 \alpha \sum_{j=1}^{n-1} r_{j} U_{n-j}+4 \rho \sum_{j=1}^{n-1} r_{j} V_{n-j} .
$$

Thus, we get, with the help of equations (22),

$(n+1)\left|p_{n+1}\right| \leq k_{n} \epsilon^{n}\left(\bar{r}+L_{z}^{2} \bar{R}\right)+k_{n} \epsilon^{n}\left(4 \alpha\left(\left|r_{0}\right| \bar{U}+\bar{r}\left|U_{0}\right|\right)+4 \rho\left(\left|r_{0}\right| \bar{V}+\bar{r}\left|V_{0}\right|\right)\right)+\epsilon^{n}(4 \alpha \bar{r} \bar{U}+4 \rho \bar{r} \bar{V}) \sum_{j=1}^{n-1} k_{j} k_{n-j}$.

Therefore, we deduce that a sufficient condition for (28) is the inequality

$$
k_{n}\left(\bar{r}+L_{z}^{2} \bar{R}+4 \alpha\left(\left|r_{0}\right| \bar{U}+\bar{r}\left|U_{0}\right|\right)+4 \rho\left(\left|r_{0}\right| \bar{V}+\bar{r}\left|V_{0}\right|\right)\right)+(4 \alpha \bar{r} \bar{U}+4 \rho \bar{r} \bar{V}) \sum_{j=1}^{n-1} k_{j} k_{n-j} \leq(n+1) k_{n+1} \epsilon \bar{p} .
$$

The identity

$$
n+1=(j+1)(n-j+1)-j(n-j)
$$

implies that

and then

$$
(n+1) k_{j} k_{n-j}=\frac{1}{n}\left(\frac{1}{j}+\frac{1}{n-j}\right)-\frac{1}{n+2}\left(\frac{1}{j+1}+\frac{1}{n-j+1}\right)
$$

$$
(n+1) \sum_{j=1}^{n-1} k_{j} k_{n-j}=\frac{2\left(n-1+2 \sigma_{n-1}\right)}{n(n+2)} \text {. }
$$

However,

which implies that

$$
\sigma_{n-1} \leq n-1
$$

Therefore, a sufficient condition for 28 is

$$
\frac{2\left(n-1+2 \sigma_{n-1}\right)}{n} \leq 3\left(1-\frac{1}{n}\right)<3 .
$$

$$
\frac{2}{3}\left(\bar{r}+L_{z}^{2} \bar{R}+4 \alpha\left(\left|r_{0}\right| \bar{U}+\bar{r}\left|U_{0}\right|\right)+4 \rho\left(\left|r_{0}\right| \bar{V}+\bar{r}\left|V_{0}\right|\right)\right)+3(4 \alpha \bar{r} \bar{U}+4 \rho \bar{r} \bar{V}) \leq \epsilon \bar{p} .
$$

By symmetry from (21), a sufficient condition for 29) is that

$$
\frac{2}{3}\left(\bar{z}+4 \alpha\left(\left|z_{0}\right| \bar{V}+\bar{z}\left|V_{0}\right|\right)+4 \rho\left(\left|z_{0}\right| \bar{U}+\bar{z}\left|U_{0}\right|\right)\right)+3(4 \alpha \bar{z} \bar{V}+4 \rho \bar{z} \bar{U}) \leq \epsilon \bar{q} .
$$

At this point, we have derived sufficient conditions in order that the inequalities should be fulfilled recurrently. Now, it rest to check their compatibility. As stated by Deprit [14], $\epsilon$ can always be chosen so large that (30), (31), (38), and (39) are satisfied no matter what values the constants possess. Also, it follows from (34) that we must choose

$$
\bar{r}<\frac{3}{20} r_{0},
$$

after which (34) is satified provided that we choose $\bar{R}$ sufficiently large. After this, (36) and (37) will be satisfied, by taking $\bar{r}$ and $\bar{z}$ sufficiently small with respect to $\bar{U}$ and $\bar{V}$. Taking small values for $\bar{r}$ and $\bar{z}$ should be compensated by choosing $\epsilon$ sufficiently large.

This article is protected by copyright. All rights reserved. 
If inequalities (23)-29) are satisfied for any $n \geq 2$, then the recurrent series obtained as solution to (15)-(21) are dominated each by series of the form

$$
A+B t+C \sum_{n \geq 2} k_{n} \epsilon^{n} t^{n}
$$

which converges in the disk $|t|<1 / \epsilon$. Thus, the series 15-21) are convergent in this disk.

\section{I EXAMINATION OF THE METHOD}

In this section, we present a comparison of the integration by recurrent power series (RPS) against the Dormand-Prince method (DOPRI5) [18]. We have performed the integration of the problem for $\omega=\mu=1, \beta=-1.2, \alpha=0.2, L_{z}=0.1, H=0.32$, and with initial conditions

$$
r_{0}=0.128, \quad z_{0}=0, \quad \dot{r}_{0}=-0.064, \quad \dot{z}_{0}=0.096311
$$

along several periods of time, from $T=10$ to $T=200$ units of time.

Table 1 includes the computational time required to compute the numerical solution along the time period $T$ by using DOPRI5 and RPS. The accuracy of both methods has been fixed to $\epsilon=10^{-17}$. In the RPS solution, we have taken $M=20$. We have included in Table 2 the value of the energy constant $H$ computed at $T=10,100,200,400,600,800,1000$ to check the accuracy of the numerical solution. All the computations have been performed by using a $2.53 \mathrm{GHz}$ Intel Core 2 Duo processor.

Comparing the results shown in Table 1 we notice that RPS is about 1000 times faster than DOPRI5. The energy constant associated with both numerical solutions remained the same to 15 significant figures, as it is shown in Table 2 In Figure 4 , we show the evolution of the step size $h$ along the period of integration $T=100$, for DOPRI5 and RPS, for an accuracy of $\epsilon=10^{-17}$.

Table 3 includes the absolute value of the difference in $r$ and $z$ of the numerical solution computed by using DOPRI5 at several values of $T$, from $T=10$ to $T=200$, with respect to the RPS solution. Let $r_{R}(T), z_{R}(T)$ be the value of the numerical solution computed through the RPS method at time $T$, and $r_{D}(T), z_{D}(T)$ the numerical solution computed by using DOPRI5, at the same values of the integration time $T$. In Table 3, we show the quantities $r_{R}(T), r_{D}(T),\left|r_{R}(T)-r_{D}(T)\right|$ and $\left|z_{R}(T)-z_{D}(T)\right|$ at each value of $T$. We observe that there are significative differences, of the order of $10^{-8}$, between both numerical solutions at $T=100$.

Zotos [12] studied the nature of motion (regular and chaotic) in this galactic system for values of the energy larger than the energy of escape, finding that there are two kind of chaotic orbits. The first type consists of chaotic orbits that remain trapped inside the curves of zero velocity for long intervals of time before escaping to infinity. The second type of chaotic orbits consists of chaotic orbits that escape from the curves of zero velocity in very short intervals of time. As the system we are dealing with is chaotic, each initial condition is arbitrarily close to other initial conditions with significantly different evolution in time. A deviation of the order of $10^{-8}$ in the numerical solution leads to a non reliable numerical solution if the equations of motion are integrated over long spans of time.

In Table 4 we compare the numerical solution RPS, calculated with an accuracy of $\epsilon=10^{-17}$, with the numerical solution RPS fixing the accuracy to $\epsilon_{1}=10^{-21}$. Let $r(T), z(T)$ and $r_{1}(T), z_{1}(T)$ be the numerical solutions computed at time $T$ with accuracy $\epsilon=10^{-17}$ and $\epsilon_{1}=10^{-21}$, respectively. Table 4 includes the values of $r(T),\left|r(T)-r_{1}(T)\right|, z(T)$ and $\left|z(T)-z_{1}(T)\right|$, for several values of $T$, from $T=100$ to $T=200$. Table 5 shows the results of the same comparison for DOPRI5. In these tables, we observe that the difference between the RPS numerical solutions at $T=100$ is about $10^{-10}$, while the difference between the DOPRI5 numerical solutions is of the order of $10^{-8}$. The deviations between the numerical solutions at $T=140$ is about $10^{-7}$ and $10^{-5}$ for the RPS and DOPRI5 solutions, respectively. Thus, we can conclude that the numerical solution computed with the RPS method is more accurate than the DOPRI5 numerical solution by a factor of 100. For intervals of integration larger than $T=180$, the deviations between the numerical solutions are of the same order than the solution itself, so we can consider that these numerical solutions are not reliable for such values of $T$.

Backward error analysis is a very useful tool for the study of the long-time numerical behavior of numerical integrators. In order to show the validity of the numerical solutions, we have integrated backward the equations of motion of the system taking the result of the numerical integration of the problem for $T=100$ and $T=140$ as initial conditions. Let $r_{R}(T), z_{R}(T), \dot{r}_{R}(T), \dot{z}_{R}(T)$ and $r_{D}(T), z_{D}(T), \dot{r}_{D}(T), \dot{z}_{D}(T)$ be the numerical solutions obtained at time $T$ by RPS and DOPRI5, respectively, fixing the accuracy to $\epsilon=10^{-21}$, and taking

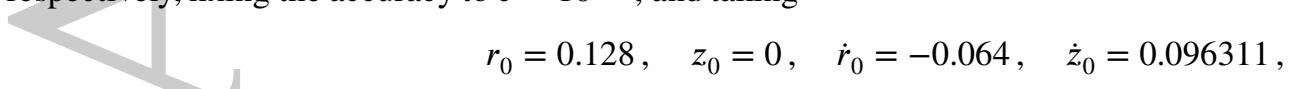

This article is protected by copyright. All rights reserved. 
as initial conditions given at $T=0$. In Table 6 , we show the result of the backward integration of the the equations of motion by taking as initial conditions $r_{R}(T), z_{R}(T), \dot{r}_{R}(T), \dot{z}_{R}(T)$, with $T=100$ and $T=140$, respectively. In the last column, we have included the value of the energy constant computed at $T=0$. The results of the backward integration of the equations of motion by taking as initial conditions $r_{D}(T), z_{D}(T), \dot{r}_{D}(T), \dot{z}_{D}(T)$, with $T=100$ and $T=140$, are shown in Table 7 In both tables, we have included the difference between the initial conditions $r_{0}$ and $z_{0}$ and the corresponding numerical approximation obtained after computing backward the solution with initial conditions $\left(r_{R}(T), z_{R}(T), \dot{r}_{R}(T), \dot{z}_{R}(T)\right)$ and $\left(r_{D}(T), z_{D}(T), \dot{r}_{D}(T), \dot{z}_{D}(T)\right)$.

We can conclude that the deviation obtained in the initial conditions at $T=0$ as a result of the backward integration by the RPS method is about $10^{-8}$ for $T=100$, and $10^{-4}$ for $T=140$. The deviation obtained with DOPRI5 is of the order of $10^{-6}$ for $T=100$, and $10^{-3}$ for $T=140$. Moreover, the energy constant remained the same at the end of the backward integration to 15 significant figures in the RPS numerical solution, but not with DOPRI5, presenting a deviation of the order of $10^{-13}$ with respect to $H_{0}$. This means, again, that RPS is more accurate than DOPRI5 by a factor of 100 .

\section{I CONCLUSIONS}

In this paper, we have adapted the ideas of the ideas of Deprit and Price [14] and Steffensen [15] to integrate the equations of motion of a a time-independent axially symmetric galactic-type potential, presenting only one opening to infinity, by recurrent power series. We have proved the convergence of these recurrent power series and compared the integration by recurrent power series against the Dormand-Prince method.

The numerical experiments show that the RPS method is 1000 times faster than DOPRI5. We have carried out several numerical tests in order to measure the reliability of the numerical solutions. We have checked that the energy constant associated with both numerical solutions (RPS and DOPRI5) remained the same to 15 significant figures, considering an accuracy of $\epsilon=10^{-17}$ in the numerical solutions. Moreover, we have integrated the equations of motion for two values of the accuracy to analyze the deviation between both numerical solutions for each numerical method, and performed a backward error analysis to check the reliability of the numerical solutions. Taking into account all the numerical tests, we can conclude that the numerical solution computed via RPS is more accurate than the DOPRI5 numerical solution by a factor of 100 .

\section{Conflict of interest}

The author declares no potential conflict of interests.

\section{REFERENCES}

1. Aguirre J, Vallejo JC, Sanjuan MAF. Wada basins and chaotic invariant sets in the Hénon-Heiles system. Phys. Rev. E 2001; 64 (11): 066208. DOI: 10.1103/PhysRevE.64.066208.

2. Aguirre J, Sanjuan MAF. Limit of small exits in open Hamiltonian systems. Phys. Rev. E 2003; 67: 056201. DOI: 10.1103/PhysRevE.67.056201.

3. Barbanis B. Escape regions of a quartic potential. Celest. Mech. Dyn. Astron. 1990; 48 (1): 57-77. DOI: 10.1007/BF00050676.

4. Barrio R, Blesa F, Serrano S. Bifurcations and safe regions in open Hamiltonians. New Journal of Physics 2009; 11: 053004. DOI: 10.1088/1367-2630/11/5/053004.

5. Blesa F, Seoane JM, Barrio R, Sanjuan MAF. To escape or not to escape, that is the question - Perturbing the Hénon-Heiles Hamiltonian. Int. J. Bifurcat. Chaos 2012; 22 (6): 1230010. DOI: 10.1142/S0218127412300108.

6. Contopoulos G. Asymptotic curves and escapes in Hamiltonian systems. Astron. Astrophys. 1990; 231 (1): 41-45.

7. Contopoulos G, Kaufmann D. Types of escapes in a simple Hamiltonian system. Astron. Astrophys. 1992; 253 (2): 379 388 . 
8. de Moura APS, Letelier PS. Fractal basins in Hénon-Heiles and other polynomial potentials. Phys. Lett. A 1999; 256: 362-368. DOI: 10.1016/S0375-9601(99)00209-1.

9. Siopsis C, Kandrup HE, Contopoulos G, Dvorak R. Universal properties of escape in dynamical systems. Celest. Mech. Dyn. Astron. 1996; 65(1-2): 57-68. DOI: 10.1007/BF00048438.

10. Navarro JF, Henrard J. Spiral windows for escaping stars. Astron. Astrophys. 2001; 369: 1112-1121. DOI: 10.1051/00046361:20010166.

11. Navarro JF. Windows for escaping particles in quartic galactic potentials. Appl. Math. Comp. 2017; 303: 190-202. DOI: 10.1016/j.amc.2017.01.040.

12. Zotos EE. Trapped and escaping orbits in an axially symmetric galactic-type potential. PASA 2012; 29: 161-173. DOI: 10.1071/AS12008.

13. Zotos EE. Escape dynamics in a Hamiltonian system with four exit channels. Nonlinear Studies 2015; 22 (3): 1-20.

14. Deprit A, Price JF. The computation of characteristics exponents in the planar restricted problem of three bodies. The Astr. Journal 1965; 70 (10): 836-846.

15. Steffensen JF. On the restricted problem of three bodies. Kgl. Danske Videnskab. Selskab, Mat. Fys. Medd. 1956; 30 (18): 17.

16. Deprit A, Price JF. Numerical integration by recurrent power series. Astron. Astrophys. 1969; 1: 427.

17. Navarro JF. Numerical integration of the $N$-body ring problem by recurrent power series. Celest. Mech. Dyn. Astr. 2018; 130 (2): 16. DOI: 0.1007/s10569-018-9816-X.

18. Hairer E, Norsett SP, Wanner G. Solving ordinary differential equations I. Nonstiff problems. Berlin. Springer. 2008.

19. Caranicolas ND, Vargoglis H. Families of periodic orbits in a quartic potential. Astron. Astrophys. 1984; 141: 383-388.

20. Deprit A, Elipe A. The Lissajous transformation II. Normalization. Celest. Mech. Dyn. Astron. 1991; 51: 227.

21. Elipe A, Deprit A. Oscillators in resonance. MRC, 1999; 26: 635.

22. Sitarski G. Recurrent power series integration of the equations of comet's motion. Acta Astronomica 1979; 29: 401-411.

\section{AUTHOR BIOGRAPHY}

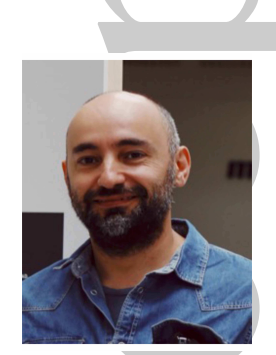

Juan F. Navarro completed his PhD degree from the University of Alicante, Alicante, Spain, in 2002. He is a recipient of the Extraordinary Award of the University of Alicante for his master thesis on the rotation of the rigid Earth. This work took part in the project Pinpoint positioning in a wobbly world awarded with the Descartes Prize in 2003, an annual award in science given by the European Union to outstanding scientific achievements resulting from European collaborative research. He is currently Professor at the Department of Applied Mathematics, University of Alicante, member of the Scientific Group on Space Geodesy and Space Dynamics of the University of Alicante, and member of the International Astronomical Union. His scientific interests include different problems in Celestial Mechanics, such as the study of the rotational motion of the Earth, the escape of particles from galactic potentials, and the numerical exploration of the $N$-body ring problem.

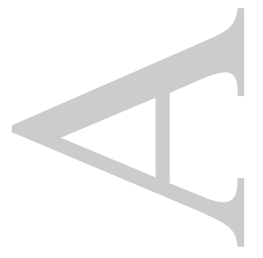

This article is protected by copyright. All rights reserved. 


\begin{tabular}{rrr}
\hline$T$ & RPS CPU time $(\mathrm{ms})$ & DOPRI5 CPU time $(\mathrm{ms})$ \\
\hline 10 & 1.073 & 268.920 \\
20 & 1.829 & 560.411 \\
30 & 2.502 & 812.070 \\
40 & 3.645 & 1088.251 \\
50 & 4.188 & 1381.029 \\
60 & 4.980 & 1676.946 \\
70 & 5.936 & 2060.591 \\
80 & 7.085 & 2271.016 \\
90 & 7.827 & 2526.549 \\
100 & 9.403 & 2833.502 \\
\hline
\end{tabular}

TABLE 1 Computational time required to compute the numerical solution along the time interval $T=$ $10,20,30,40,50,60,70,80,90,100$ by using RPS and DOPRI5. The accuracy of the numerical solutions has been fixed to $\epsilon=10^{-17}$.

\begin{tabular}{rrr}
\hline$T$ & $H(T)$ with RPS & $H(T)$ with DOPRI5 \\
\hline 10 & 0.32000000000000 & 0.32000000000000 \\
100 & 0.32000000000000 & 0.32000000000000 \\
200 & 0.32000000000000 & 0.32000000000000 \\
400 & 0.32000000000000 & 0.32000000000000 \\
600 & 0.32000000000000 & 0.32000000000000 \\
800 & 0.32000000000000 & 0.32000000000000 \\
1000 & 0.32000000000000 & 0.32000000000000 \\
\hline
\end{tabular}

TABLE 2 Energy constant computed at $T=10,100,200,400,600,800,1000$ by using RPS and DOPRI5. The accuracy of the numerical solutions has been fixed to $\epsilon=10^{-17}$.

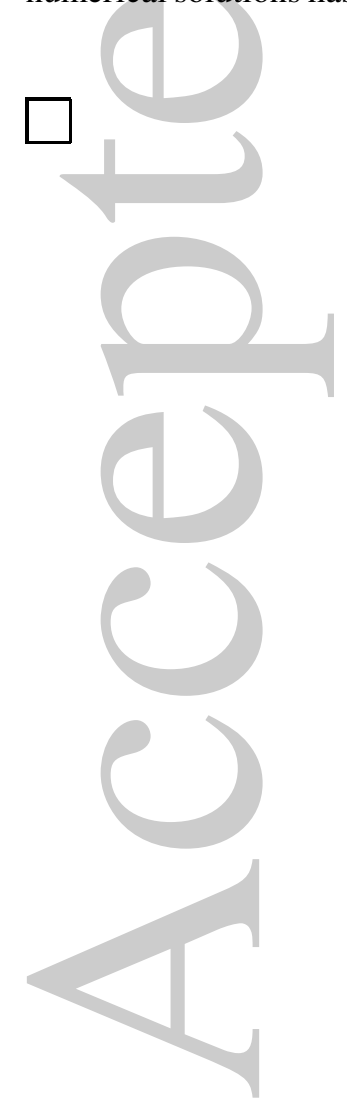

This article is protected by copyright. All rights reserved. 


\begin{tabular}{rrrrr}
\multicolumn{7}{c}{} & & & & \\
\hline$T$ & & $r_{R}(T)$ & & \\
& & & & \\
\hline 10 & 0.36958648425428 & 0.36958648425429 & 0.00000000000001 & 0.00000000000000 \\
20 & 0.19808731178258 & 0.19808731178255 & 0.00000000000003 & 0.00000000000001 \\
30 & 0.66285608865412 & 0.66285608865419 & 0.00000000000007 & 0.00000000000005 \\
40 & 0.77214314226319 & 0.77214314226514 & 0.00000000000195 & 0.00000000000058 \\
50 & 0.93746128111784 & 0.93746128111407 & 0.00000000000377 & 0.00000000000236 \\
60 & 0.69968897674027 & 0.69968897670430 & 0.00000000003597 & 0.00000000000672 \\
70 & 0.54348508787008 & 0.54348508807995 & 0.00000000020987 & 0.00000000019449 \\
80 & 0.19722195658053 & 0.19722195982284 & 0.00000000324231 & 0.00000000161651 \\
90 & 0.54636876217503 & 0.54636877144641 & 0.00000000927138 & 0.00000000256142 \\
100 & 0.41769314053241 & 0.41769311294220 & 0.00000002759021 & 0.00000000652755 \\
120 & 0.64577635466334 & 0.64577669783707 & 0.00000034317373 & 0.00000007444115 \\
140 & 0.42966098990418 & 0.42962817963248 & 0.00003281027170 & 0.00000516826968 \\
160 & 0.82356022732990 & 0.82174463500055 & 0.00181559232935 & 0.00127321899156 \\
180 & 0.41939367765439 & 0.48278151641576 & 0.06338783876137 & 0.07585464999168 \\
200 & 0.70856351474134 & 0.43766996572273 & 0.27089354901861 & 0.16555383692506 \\
\hline
\end{tabular}

TABLE 3 Difference in $r$ and $z$ of the numerical solution computed by using DOPRI5 at time $T=$ $10,20,30,40,50,60,70,80,90,100,120,140,160,180,200$, with respect to the RPS numerical solution. The numerical solutions have been computed fixing the accuracy to $\epsilon=10^{-17}$.

\begin{tabular}{|c|c|c|c|c|}
\hline & $r(T)$ & $\left|r(T)-r_{1}(T)\right|$ & $z(T)$ & $\left|z(T)-z_{1}(T)\right|$ \\
\hline 100 & 0.41769314053241 & 0.00000000059664 & -0.24040934361301 & 0.00000000014116 \\
\hline 120 & 0.64577635466334 & 0.00000000742120 & -0.12315938274744 & 0.00000000160980 \\
\hline 140 & 0.42966098990418 & 0.00000070968679 & -0.31341725691129 & 0.00000011183821 \\
\hline 160 & 0.82356022732990 & 0.00003846950082 & 0.17470790698460 & 0.00002725202588 \\
\hline 180 & 0.41939367765439 & 0.00376644913568 & -0.24969111466002 & 0.00461132506109 \\
\hline 200 & 0.70856351474134 & 0.21685835094132 & 0.23554437285294 & 0.11722017799742 \\
\hline
\end{tabular}

TABLE 4 Difference in $r$ and $z$ of the numerical solution computed by using RPS with an accuracy of $\epsilon=10^{-17}$ at times $T=100,120,140,160,180,200$, with respect to the RPS numerical solution computed with an accuracy of $\epsilon_{1}=10^{-21}$.

\begin{tabular}{|c|c|c|c|c|}
\hline$T$ & $r(T)$ & $\left|r(T)-r_{1}(T)\right|$ & $z(T)$ & $\left|z(T)-z_{1}(T)\right|$ \\
\hline 100 & 0.41769311294220 & 0.00000002748450 & -0.24040933708546 & 0.00000000650254 \\
\hline 120 & 0.64577669783707 & 0.00000034185888 & -0.12315945718859 & 0.00000007415593 \\
\hline 140 & 0.42962817963248 & 0.00003268453457 & -0.31342242518097 & 0.00000514845521 \\
\hline 160 & 0.82174463500055 & 0.00180877306375 & 0.17598112597616 & 0.00126838940064 \\
\hline 180 & 0.48278151641576 & 0.06275629395118 & -0.32554576465170 & 0.07675451497025 \\
\hline 200 & 0.43766996572273 & 0.26917103648764 & 0.06999053592788 & 0.15509962313563 \\
\hline
\end{tabular}

TABLE 5 Difference in $r$ and $z$ of the numerical solution computed by using DOPRI5 with an accuracy of $\epsilon=10^{-17}$ at times $T=100,120,140,160,180,200$, with respect to the DOPRI5 numerical solution computed with an accuracy of $\epsilon_{1}=10^{-21}$.

This article is protected by copyright. All rights reserved. 


\begin{tabular}{rrrrrrrr}
\hline$T$ & $r_{0}$ & $r_{R}(0)$ & $\left|r_{0}-r_{R}(0)\right|$ & $z_{0}$ & $z_{R}$ & $\left|z_{0}-z_{R}(0)\right|$ & $H_{R}(0)$ \\
\hline 100 & 0.128 & 0.12800003969894 & 0.00000003969894 & 0 & 0.00000015267959 & 0.00000015267959 & 0.32000000000000 \\
140 & 0.128 & 0.12812628919959 & 0.00012628919959 & 0 & 0.00045402398509 & 0.00045402398509 & 0.32000000000000 \\
\hline
\end{tabular}

TABLE 6 Backward integration of the equations of motion with RPS by taking as initial conditions $r_{R}(T), z_{R}(T), \dot{r}_{R}(T), \dot{z}_{R}(T)$, with $T=100$ and $T=140$. The energy remains the same to 15 significant figures.

\begin{tabular}{rrrrrrrrr} 
& & & & & \\
\hline$T^{\prime}$ & $r_{0}$ & $r_{D}(0)$ & $\left|r_{0}-r_{D}(0)\right|$ & $z_{0}$ & $z_{D}$ & $\left|z_{0}-z_{D}(0)\right|$ & & $H_{D}(0)$ \\
& & & & & & & \\
\hline 100 & 0.128 & 0.12800171977632 & 0.00000171977632 & 0 & 0.00000660757500 & 0.00000660757500 & 0.32000000000031 \\
140 & 0.128 & 0.12858803913348 & 0.00058803913348 & 0 & -0.00814829850157 & 0.00814829850157 & 0.32000000000049 \\
\hline
\end{tabular}

TABLE 7 Backward integration of the equations of motion with DOPRI5 by taking as initial conditions $r_{D}(T), z_{D}(T), \dot{r}_{D}(T), \dot{z}_{D}(T)$, with $T=100$ and $T=140$. The energy presents a deviation from its original value $H_{0}=0.32$.

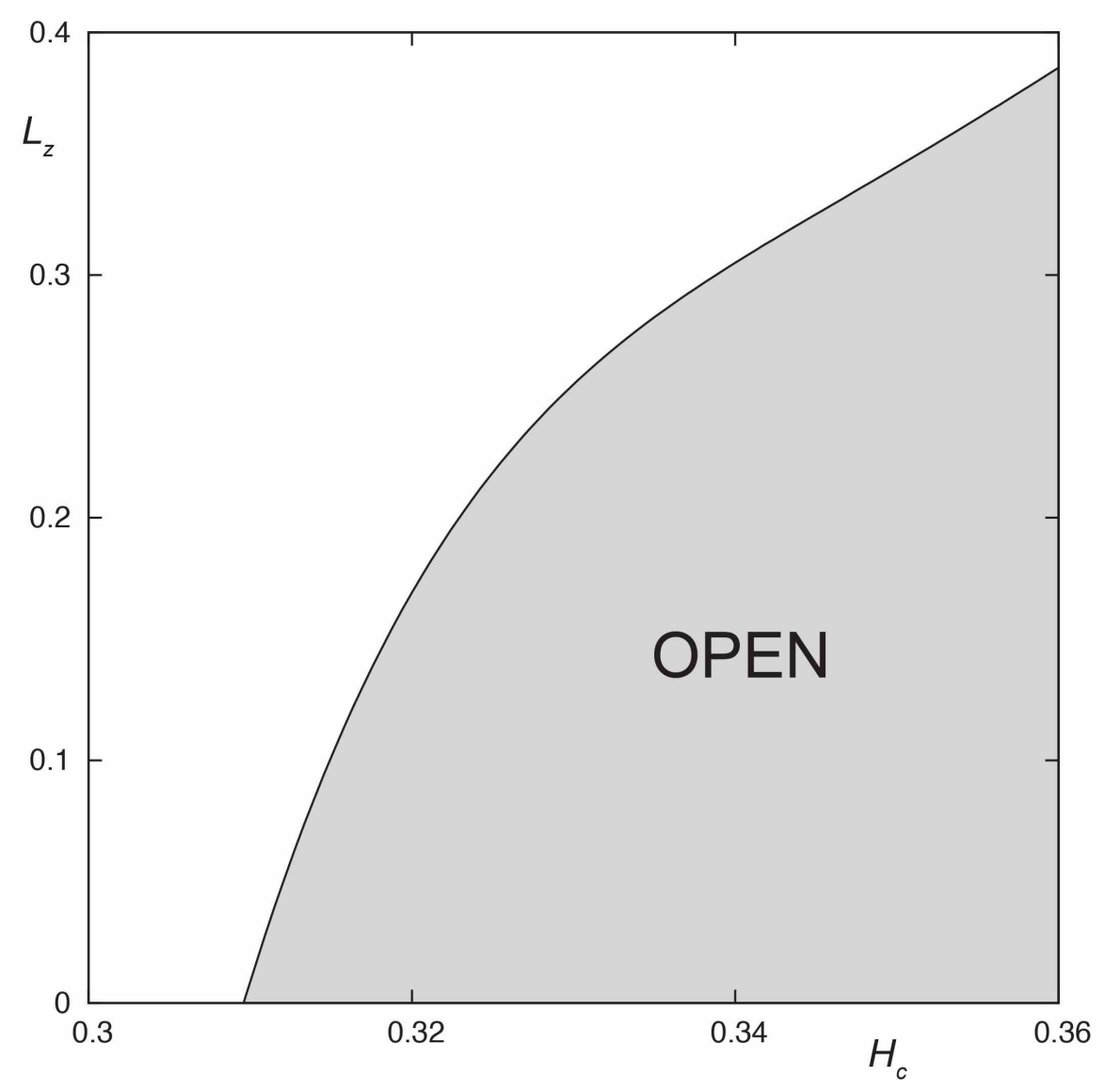

FIGURE 1 Relation between the energy of escape $H_{c}$ of a test particle and $L_{z}$. In the region in gray, the curves of zero velocity are open, and test particles can escape from the potential well.

This article is protected by copyright. All rights reserved. 

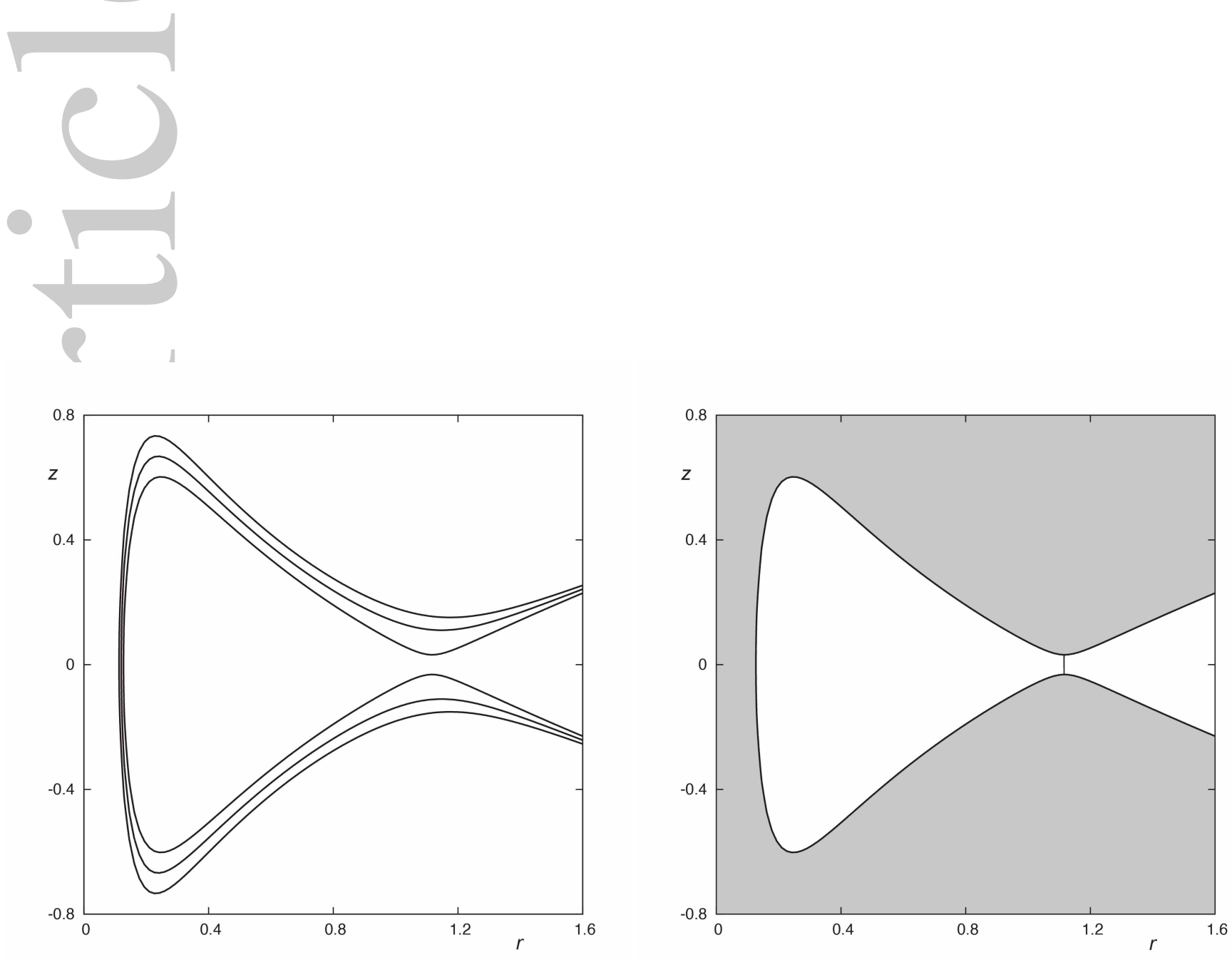

FIGURE 2 Curves of zero velocity for $\omega=1, \mu=1, \alpha=0.2, \rho=-1.2, L_{z}=0.1$ and different values of the energy larger than the critical value: $H_{1}=0.32, H_{2}=0.36$ and $H_{3}=0.4$ (left panel). As the energy of the system grows, the windows of the potential well become wider. 

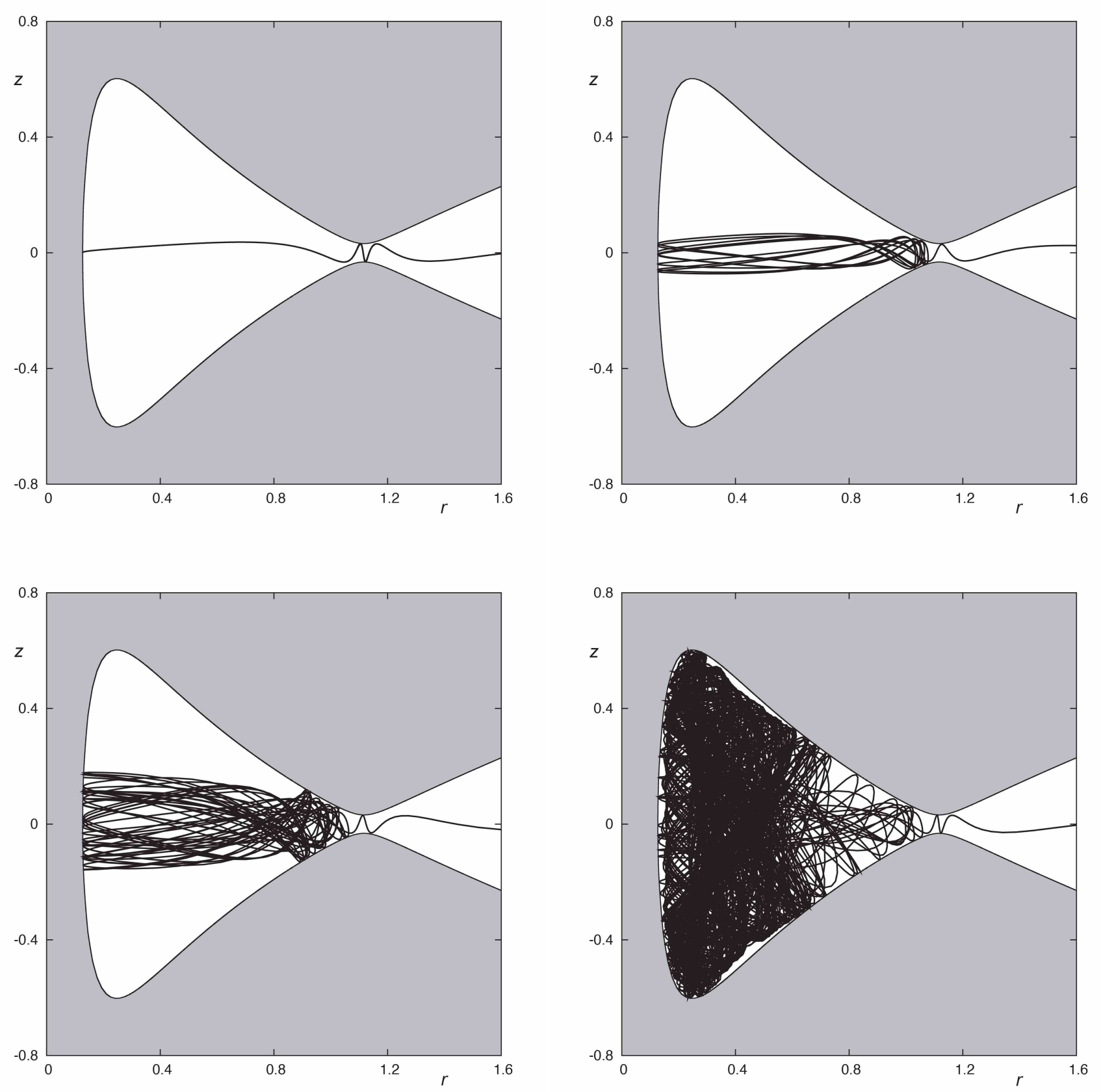

FIGURE 3 Examples of orbits with different escaping times $t_{e}$ for $\omega=1, \mu=1, \alpha=0.2, \rho=-1.2$ and $L_{z}=0.1: t_{e}<10$ (upper-left panel), $10<t_{e}<100$ (upper-right panel), $100<t_{e}<500$ (lower-left panel) and $500<t_{e}<1000$ (lower-right panel). 


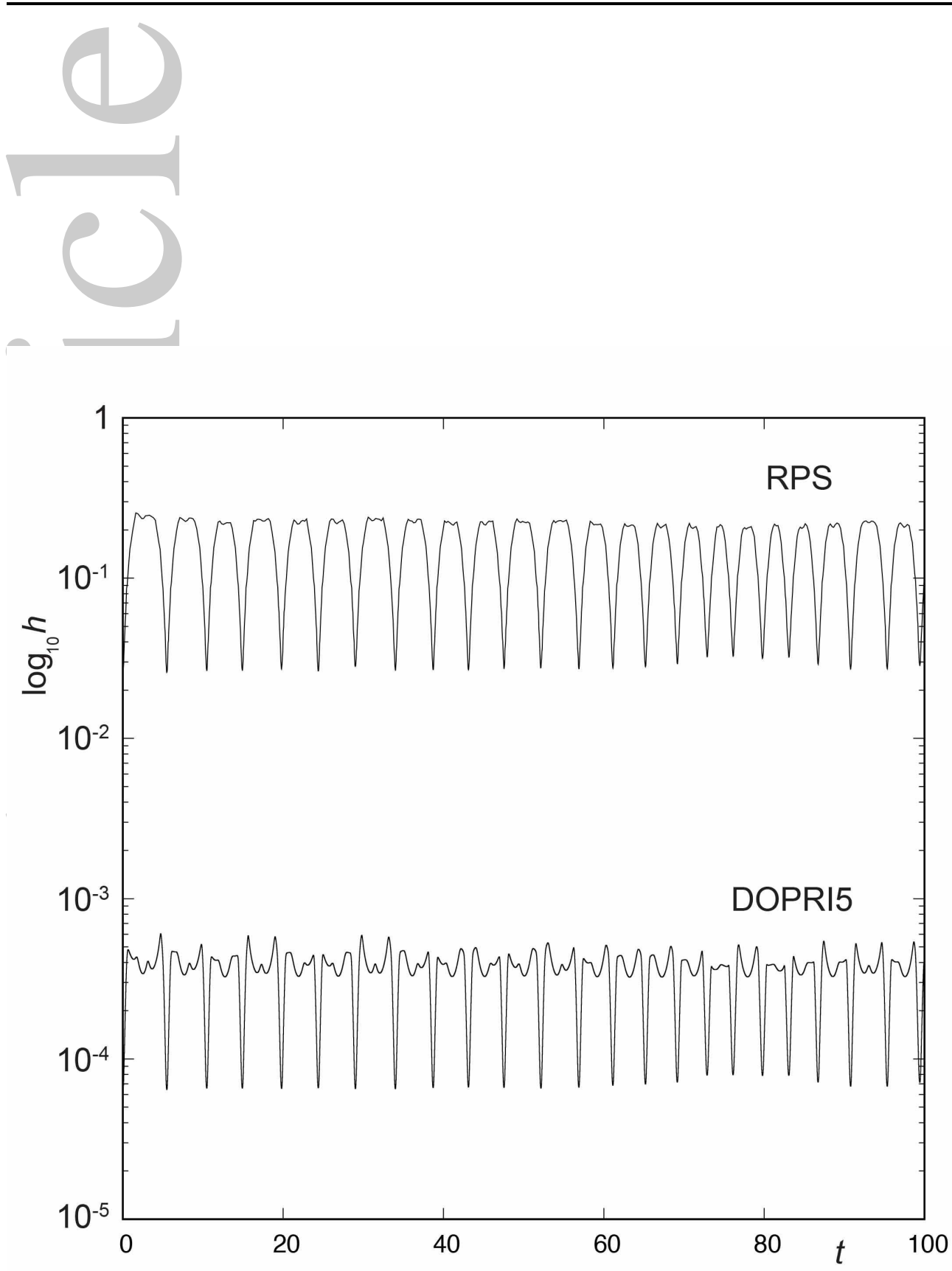

FIGURE 4 Comparison of $\log _{10} h$ with respect to the integration time $t$, from $t=0$ to $t=100$, for RPS and DOPRI5. The accuracy of the numerical solutions has been fixed to $\epsilon=10^{-17}$.

This article is protected by copyright. All rights reserved. 